\title{
Exploring the Multi-minima Behavior of Small Molecule Crystal Polymorphs at Finite Temperature
}

\author{
Eric C. Dybeck, ${ }^{\dagger}$ David P. McMahon ${ }^{\ddagger}$ Graeme M. Day, ${ }^{\ddagger}$ and Michael R. \\ Shirts* \\ $\dagger$ Department of Chemical Engineering, University of Virginia, Charlottesville, Virginia \\ 22904, USA \\ $\ddagger$ School of Chemistry, University of Southampton, Southampton, UK \\ IDepartment of Chemical and Biological Engineering, University of Colorado Boulder, \\ Boulder, CO 80309, USA \\ E-mail: michael.shirts@colorado.edu
}

\begin{abstract}
The predicted ambient-temperature crystal structures of organic small molecules are often represented by a single energy-minimized configuration at zero Kelvin. This procedure effectively collapses the ensemble of configurations that would be present at ambient temperature into a single representative structure. This simplification is likely to break down if the crystal structure has multiple different lattice energy minima within the ambient temperature ensemble. In this paper, we explore the existence of multiple minima within finite-temperature crystal basins by sampling crystals at a range of temperatures followed by rapidly quenching the configurations. We then observe whether each crystal returns to the original minimum
\end{abstract}


or to an ensemble of minima on the lattice energy landscape. Eight of the twelve compounds examined in this work have at least one polymorph with multi-minima behavior. These multi-minima basins have implications for crystal structure prediction studies, and it is therefore important to understand the factors that lead a crystal structure to have multiple minima. We find that in general, the existence of multiple minima within a crystal basin is more likely for the compounds that are larger and have more flexibility. We find that the number of minima found tends to increase with the sampling temperature, and the lattice energy of minima found from the same finite-temperature trajectory can vary by $>2.0 \mathrm{~kJ} / \mathrm{mol}$. Finally, we show that the lattice energy minima contained within a single ambient temperature basin can have different space groups and numbers of molecules in the asymmetric unit. Overall, the data suggest that many experimental crystal polymorphs are highly likely to have multi-minima behavior and are best described by an ensemble of structures encompassing many minima rather than by a single lattice-energy minimum.

\section{Introduction}

The a priori prediction of crystal structures from only molecular connectivity or 3D gas phase structure is an ongoing challenge in modern solid state-chemistry. ${ }^{1,2}$ Knowing the solid forms of an organic molecule enables the possibility to predict or engineer critical solid form

properties such as solubility, ${ }^{3}$ chemical stability ${ }^{4}$ and particle morphology ${ }^{5,6}$ to streamline commercial development. As a prime example, early knowledge of the thermodynamically stable solid form can reduce the instances of late-stage transformations delaying the release of innovative pharmaceutical products. ${ }^{7-9}$

The most widely used methodology for computational crystal structure prediction (CSP) generates candidate structures by identifying minima on the lattice energy surface. ${ }^{10}$ In this approach, the energy-minimized structure with the lowest energy and all other minima within a modest energy window above the global minimum (usually around 10-15 kJ/mol) ${ }^{11}$ 
are considered to be the potentially observable polymorphs under experimental conditions.

Many researchers have noted that this lattice energy minima approach to predicting real structures may be limited by the fact that it fundamentally operates at $0 \mathrm{~K}$ rather than at room temperature, where the actual crystallization experiments are performed and properties are of most interest. ${ }^{12-16}$ Nevertheless, this method will work well at predicting the experimental crystal structure in systems that satisfy certain underlying assumptions. These assumptions are that 1) each lattice energy minimum corresponds with a unique ambient temperature solid form, and that 2) the relative stability of the solid form at ambient temperature can be approximated by the energy difference between the corresponding $0 \mathrm{~K}$ minimum and the $0 \mathrm{~K}$ minima of other putative solid forms. The recent success of these lattice-energy minima approaches in the blind tests of organic crystal structure prediction suggests that these assumptions are satisfied for the small rigid molecules typically used in the blind tests. ${ }^{17-19}$ However, the assumption of an accurate and unambiguous mapping between $0 \mathrm{~K}$ lattice energy minima and ambient temperature structures could break down in more complex molecules, and a systematic probing of this assumption on a set of compounds that span a range of size, flexibility, and disorder has not been previously performed.

An ambient-temperature crystal basin will not have a clear mapping to a $0 \mathrm{~K}$ energy minimum in cases where the basin contains more than one local minimum on the lattice energy surface. A depiction of this 'rough' basin with multiple minima is shown in Figure 1 , in contrast to the traditional picture of a smooth basin with a single unambiguous energy minimum. Predicting the stable experimental forms in compounds with rough crystal basins will be challenging with lattice energy minima-based approaches. This difficulty will occur because not all local energy minima correspond to new solid forms and because the stability assigned to the basin will depend on which of the numerous local minima within the basin is used to characterize its stability. Indeed, many researchers at the forefront of CSP have pointed to this ambiguity as being responsible for the observed trend of too many predicted stable crystal forms. ${ }^{12,20-23}$ CSP studies of small molecules can easily produce 100 s-1000s of 
lattice energy minima with similar energies. ${ }^{12,20,23-27}$ However, these same molecules typically have only 2-5 experimentally observed crystal forms. Determining which energy minima are part of the same basin, or at least understanding which compounds will have multi-minima basins, could improve the ability to identify the true stable solid forms within the crowded CSP lattice energy landscape.

One can probe the 'roughness' of a crystal basin near a local minimum by heating the corresponding structure up to finite temperature and then quenching to $0 \mathrm{~K}$ (lattice energy minimizing in both atomic-coordinate and box-vector space) a set of independently sampled configurations from that high temperature state. If the finite temperature basin around the lattice energy minimum is smooth at the sampled temperature, all quenched configurations will return to the initial $0 \mathrm{~K}$ structure. Conversely, if the lattice energy landscape is rough, the independent configurations will quench into a multitude of locally stable energy minima rather than a single global energy minimum as depicted in Figure 1.

The behavior of lattice energy minima quenching into new structures after heating was first demonstrated for acetic acid using 'short MD shakeup' simulations. ${ }^{28}$ In this study, all lattice energy minima found in a CSP were perturbed with a short 60 ps MD simulation followed by re-minimization to allow unstable minima to restructure. The investigators observed that some minima overcame small barriers upon heating and subsequently cooled into a different more stable minima, consistent with them existing in basins with multiple local minima. Metadynamics studies of benzene, ${ }^{29}$ 5-fluorouracil, ${ }^{16}$ and the pigment PR $179^{30}$ have also shown that multiple different lattice energy minima can exist within an ambient temperature crystal ensemble.

However, there are lingering questions about the overall nature of crystalline systems that have rough basins with multiple lattice energy minima in the finite-temperature ensemble. Some of these questions include:

- Is there a correlation between molecular flexibility and the existence of rough basins?

- Can multi-minima behavior be predicted purely from a description of the parent 
molecule, or does the crystal packing of the molecule have an influence on whether it will contain multiple minima?

- How many distinct lattice energy minima can exist within the same finite-temperature basin?

- How does temperature affect the number and rate of transition between minima, and what does that say about the roughness of the energy surface?

- How large of an energy difference can there be between minima within the same ambient temperature basin?

- What is the structural difference between minima from the same ambient temperature basin?

In this study, we seek to explore these questions by examining the multi-minima behavior of the crystal structures of twelve polymorphic small molecule systems (shown in Figure 2). The molecules span a range of molecular size, flexibility, and crystalline order. In addition, all of these systems have enantiotropically related polymorph pairs, meaning that the globally stable polymorph changes as a function of temperature. Enantiotropism between polymorph pairs is relatively common in organic small molecules. ${ }^{31}$ Furthermore, it is important to understand multi-minima behavior in enantiotropic systems because a temperature-mediated stability re-ranking indicates a deviation from an ideal mapping between $0 \mathrm{~K}$ energy minima and stable ambient temperature structures. Some of these crystals were also observed in a previous study to find new more stable regions of phase space upon heating. ${ }^{32}$ Therefore, these compounds represent an ideal set to explore multi-minima behavior in real organic crystals (or at least within the point-charge potential used). The roughness of each crystal basin is probed by sampling at a range of temperatures up to $300 \mathrm{~K}$ to generate sets of finitetemperature configurations. These configurations are then rapidly lattice energy minimized down to $0 \mathrm{~K}$ to see whether they quench to the original minima or instead quench into a set of new minima. 


\section{Methodology}

The twelve polymorphic systems presented in our previous work ${ }^{32}$ were used as the test systems to explore the effects of heating crystals and reminimizing. The chemical structures for these compounds are shown in Figure 2. The initial crystal structures were taken from the Cambridge Structural Database and replicated to supercells with box dimensions larger than $1.6 \mathrm{~nm}$ in each dimension. A table of all polymorphs examined as well as the CSD refcodes of the initial structures and the dimensions of the supercell is provided in Table 1 of the Supporting Information. Each of the initial crystal structures were lattice energy minimized and then equilibrated to a temperature of $10 \mathrm{~K}, 50 \mathrm{~K}, 100 \mathrm{~K}, 200 \mathrm{~K}$, and $300 \mathrm{~K}$ (except cyclopentane which was heated to $10 \mathrm{~K}, 30 \mathrm{~K}, 50 \mathrm{~K}, 70 \mathrm{~K}$, and $100 \mathrm{~K}$ due to the lower melting point) for $0.1 \mathrm{~ns}$ after which a 5 ns production NPT simulation was carried out. 100 snapshots evenly spaced from this trajectory at each temperature were then lattice energy minimized to determine the minima corresponding with that finite-temperature configuration.

We note briefly that finding new minima through exploring new regions of phase space is inherently a kinetic process, and therefore the number of minima found is limited by the length of the simulation. A sufficiently longer simulation will almost certainly find more minima, and ultimately any fixed length of simulation will be somewhat arbitrary. For example, a sufficiently short simulation will always find a single minimum, and an infinitely

long simulation would in principle find all minima regardless of temperature, even those corresponding to two different experimentally stable polymorphs. The choice of $5 \mathrm{~ns}$ here is not meant to represent an exhaustive search of the crystal phase space. Rather, the 5 ns trajectory provides a brief agitation of the system which we hold constant across the crystals so that trends of multi-minima behavior with molecular size, flexibility, and temperature can be elucidated on an equal basis. 5 ns is a relatively short timescale molecular dynamics simulation, and the emergence of multi-minima behavior even on these timescales underscores the prevalence of multiple metastable minima in these systems.

All finite-temperature molecular dynamics simulations were carried out using the GRO- 
MACS simulation package, ${ }^{33}$ version 5.0.4. The precise simulation details are described in section 3.5 of our earlier work. ${ }^{32}$ Briefly, the crystals were modeled by the OPLS-AA potential with atomic point charge electrostatics. ${ }^{34-36}$ The simulations were conducted in the NPT ensemble. Langevin dynamics (stochastic dynamics integrator implementation ${ }^{37}$ ) was used to temperature couple the system. Pressure coupling in the initial equilibration was done with the anisotropic Berendsen barostat with a time constant of 1 ps and pressure control in the production simulation was done with the anisotropic Parrinello-Rahman barostat ${ }^{38}$ with a time constant of 10 ps. Long-range intermolecular interactions including both Coulombic and Lennard-Jones dispersion were treated using particle-mesh Ewald summation with a cut-off distance of $0.8 \mathrm{~nm}$ and a Fourier spacing of $0.13 \mathrm{~nm} .{ }^{39}$ The crystal configurations sampled from the finite-temperature ensemble were quenched to $0 \mathrm{~K}$ using the XTALMIN crystal minimization subroutine in Tinker version 7.0. ${ }^{40}$ Each configuration was minimized to a RMS gradient of $0.01 \mathrm{kcal} \cdot \mathrm{mol}^{-1} \cdot \AA^{-1}$.

Minimized energies for all structures are reported as the potential energy of the minimized configuration. If one is interested in determining the thermodynamic viability of a structure based on the energy, one could subtract the energy of a reference state, such as the energy of the geometry optimized molecule in a vacuum. This reference energy will depend on multiple factors, such as the vacuum conformer used, and ultimately cancels when comparing the relative stability of multiple minima within a basin minima which is the focus of this work. Therefore, we chose to directly report the potential energy of the minimized configuration independent of the choice of reference state.

All minimized structures were clustered to remove duplicates prior to the final analysis. For all clustering comparisons, a simulated pXRD pattern (wavelength $=0.7 \AA$, maximum $\left.2 \theta=20.0^{\circ}\right)$ was generated using platon ${ }^{41}$ using the full supercell. The resulting pXRD pattern was normalized such that the area under the intensity curve equaled 1. Constrained dynamic time-warping (cdtw) using a Sakoe-Chiba band constraint on the time shift was used to compare the patterns with a maximum shift in the positions of the peaks of 10 steps 
using the Euclidean distance as the cdtw metric. Clustering was performed with a Euclidean distance threshold of 0.4 if both cells remained as P1 supercells or 3.5 if one or both cells could be symmetry reduced, respectively, with patterns below the threshold considered the same. These cutoffs were determined by manually inspecting the more flexible structures to estimate which ones would likely be considered distinct structures by practitioners. All duplicate structures identified in this manner were removed prior to constructing lattice energy-density plots.

The symmetry of each structure was determined using the symmetry wrapper of pymatgen ${ }^{42}$ around the spglib routines. ${ }^{43}$ A symmetry precision tolerance of $0.1 \AA$ was used during the symmetry analysis. For a handful of structures this led to incomplete solving of the structures; for these structures, the intermediate structure was passed through the same symmetry analysis process again. The resulting structures were compared to the initial supercells to ensure no errors.

\section{Results and Discussion}

\section{Effect of molecular structure on multi-minima behavior}

We find that many crystal basins at $300 \mathrm{~K}$ contain more than one local lattice energy minimum. Figure 3 shows the largest number of unique minima found from lattice energy minimizing 100 snapshots from the room temperature MD trajectory of a polymorph in each of the 12 molecular systems examined. At $300 \mathrm{~K}$ (100K for cyclopentane), 8 of the 12 small molecule systems exhibited multi-minima behavior. A full enumeration of the lattice energy landscapes and minima for each structure is included in the Supporting Information section 2.

The number of unique minima found from quenching the 100 snapshots at finite temperature in the 12 systems tends to fall in to one of three clusters: exactly 1 minimum, 2-5 minima, and 50-100 minima. Based on the structures of the molecules in each of these three 
categories, we can draw overall conclusions about the likelihood of multi-minima behavior in molecular crystals. The systems that found 50-100 minima at $300 \mathrm{~K}$ are aripiprazole, tolbutamide, chlorpropamide, succinonitrile, cyclopentane $(100 \mathrm{~K})$. As an example of this behavior, the lattice energy landscape for chlorpropamide $\alpha$ is shown in Figure 4a including the 66 unique structures that were found from the $300 \mathrm{~K}$ trajectory. The first three molecules in this category which found 50-100 minima after quenching are larger and more flexible than the other molecules in the study. The other two molecules, cyclopentane and succinonitrile, have the distinguishing feature that one of the two crystal structures is known to be dynamically disordered. ${ }^{44,45}$ Thus we conclude that flexibility and the propensity for dynamic disorder are indicators that a large number of minima will be present in the ambient temperature basin. It is reasonable to expect that crystals with multiple minima will be precisely those which have many degrees of freedom, either internally or within the crystal, because the additional dimensions in the lattice energy surface create the opportunity for multiple local energy minima to emerge.

Many of the molecular systems in this study found exactly 1 minimum upon quenching to $0 \mathrm{~K}$ in all of the polymorphs examined. These molecules are adenine, resorcinol, paracetamol, and carbamazepine. The energy-density landscape for carbamazepine is shown in Figure 5. These molecules that always found the original minima upon quenching are distinguishable from the molecules that found 50-100 minima in that they tend to be smaller and more rigid. This aligns with the chemical intuition that molecules with fewer degrees of freedom will have smoother basins that are more likely to quench into a single minimum in contrast to molecules with multiple degrees of freedom where many local lattice energy minima exist in the energy landscape. Even though short MD simulations did not find rapidly interconverting minima in these systems, generated CSP landscapes do still find many more minima than experimentally observed forms. Thus, the over-prediction of minima in these systems does not appear to be primarily from minima connected by small kinetic barriers. However, it is in theory possible that a significantly longer and /or higher temperature simulation could 
still find solid-solid interconversion between local metastable minima in these systems.

Pyrazinamide did not fall into either of the above categories and instead 2-3 minima were found for each of forms $\alpha, \beta$, and $\gamma$ and 1 minimum was found in form $\delta$. The lattice energy landscapes for each of the four pyrazinamide structures are shown in Figure 6. Pyrazinamide therefore represents an intermediate case where the crystals has enough degrees of freedom to create more than one local energy minimum, but not enough to create 50-100. The molecule pyrazinamide also illustrates that multi-minima behavior is crystalstructure-dependent rather than purely molecule-dependent. Form $\alpha$ and form $\delta$ differ only in packing orientation. However, the former structure has multiple minima in the $300 \mathrm{~K}$ basin while the latter always quenched to the original minimum.

Chlorpropamide, piracetam, and 1,4-diiodobenzene also exhibit crystal-structure-dependent numbers of minima found at ambient temperature. Chlorpropamide form $\delta$ collapsed to 5 unique lattice energy minima after quenching the $300 \mathrm{~K}$ snapshots, while form $\alpha, \beta, \gamma$, and $\epsilon$ each found 50-100 minima (Figure 4). Piracetam form I and form III always quenched down to the original minima, while form II found two additional minima after heating and quenching from $300 \mathrm{~K}$. 1,4-diiodobenzene $\beta$ also found an additional minimum after heating and quenching from $300 \mathrm{~K}$, while form $\alpha$ always quenched to the original minimum. The energy-density landscapes for piracetam and 1,4-diiodobenzene are presented in section 2 of the Supporting Information. These examples clearly demonstrate that multi-minima behavior is crystal-structure-dependent in addition to being molecule-dependent.

In the above cases where the system has 2-3 lattice energy minima within the basin, the system will often interchange between configurations that quench to two different unique minima. Figure 7 shows a time series of the minimized lattice energy for the configuration sampled at time $t$ over the course of the 5 ns simulation for pyrazinamide $\gamma$ at $300 \mathrm{~K}$. This time series shows that the instances of finding the two minima are roughly evenly spread over the first and second half of the trajectory rather than segregated in time. This demonstrates that the two lattice energy minima found in the trajectory do not result from the system 
irreversibly moving into a new basin with a new corresponding minimum at some moment in time. Rather, the system has regions of phase space that will quench into different lattice energy minima at $0 \mathrm{~K}$, and those two regions are both sampled in some proportion at equilibrium.

In theory, one could precisely determine the fraction of time that the system will spend in each of these two distinct regions of phase space by identifying an order parameter that

distinguishes the regions and computing the ratio of partition functions over each region. ${ }^{46-49}$ Such clustering and free energy analysis is outside the scope of the present study.

The existence of multiple minima in organic small molecule crystals invariable raises an important question about what differentiates a 'distinct polymorph' from 'an additional minimum of the same polymorph'. The process of assigning configurations to distinct polymorphs is an area of active research, often involving clustering within a dimensionally reduced order parameter space, ${ }^{46-48,50}$ or identifying regions separated by high kinetic barriers. ${ }^{49}$ In this work, we adopt a kinetic view of polymorphism, in which 'multiple minima of the same polymorph' are the collection of minima that are visited by a single continuous trajectory.

\section{Effect of temperature on multi-minima behavior}

The number of minima found in the finite-temperature basins of the crystal structures examined are seen to be quite sensitive to the simulation temperature. Figure 3 shows that the number of unique minima tends to increase as temperature increases. At lower temperatures, the trajectory has insufficient energy to visit local energy minima that are much higher in energy than the average system energy at that temperature, and is incapable of visiting minima that require crossing over energy barriers that are too high. In principle, one would therefore expect a sufficiently low temperature $5 \mathrm{~ns}$ simulation to always quench to a single minimum because only a small local region of phase space is explored, and the motions are effectively harmonic. At $100 \mathrm{~K}$ and above, however, the large flexible molecules as well as the disordered systems begin to exhibit multi-minima behavior where the configurations 
quenched to new minima distinct from the initial minimized configuration. Increasing from $200 \mathrm{~K}$ to $300 \mathrm{~K}$ also resulted in a subsequent increase in the number of minima observed in these systems.

The lattice energy minima found at higher temperatures are often substantially different than those found at lower temperatures. Figure 4a shows the lattice energy minima for chlorpropamide form $\alpha$ found from quenching configurations sampled at $10 \mathrm{~K}, 50 \mathrm{~K}, 100 \mathrm{~K}$, $200 \mathrm{~K}$, and $300 \mathrm{~K}$. All configurations sampled from the three lowest temperatures quenched back to the original lattice energy minimum. The phase space explored on the nanosecond timescale at $100 \mathrm{~K}$ is therefore essentially a smooth basin corresponding to only a single local energy minimum when quenched. At $200 \mathrm{~K}$, the increased energy allowed the trajectory to explore new regions of phase space that were not accessed at $100 \mathrm{~K}$ and which contain new local energy minima distinct from the one used to initiate the simulation. At $300 \mathrm{~K}$, the system further explored new portions of the lattice energy landscape which were higher in energy and also contained more lattice energy minima distinct from those found at $200 \mathrm{~K}$. In this particular crystal, the lattice energy minima found at $300 \mathrm{~K}$ were as much as 4.5 $\mathrm{kJ} / \mathrm{mol}$ higher in energy than the original lattice energy minimum. In addition, none of the 100 configurations sampled at $300 \mathrm{~K}$ minimized back to the original lattice energy minimum; instead, each quenched to a higher energy local minimum. It is possible that slower annealing, rather than rapid quenching, could be used when attempting to find the global energy minimum within the basin so as to avoid being caught in one of these numerous higherenergy local minima on the way down to the basin's global minimum.

An increase in temperature can also cause a system to move into a new region of phase space containing minima that are lower in energy than the original lattice energy minimum. The minima found at different sampling temperatures for chlorpropamide $\beta$ are shown in Figure $4 \mathrm{~b}$. At $100 \mathrm{~K}$ and below, the sampled configurations returned to the original minimum after quenching, similar to form $\alpha$ discussed above. However, at $200 \mathrm{~K}$ a set of minima lower in energy than the original minimized configuration were found after quenching the finite- 
temperature snapshots. In this case, the minima were $1.2 \mathrm{~kJ} / \mathrm{mol}$ lower in energy than the original minimum. When the system temperature was further increased to $300 \mathrm{~K}$, another set of minima was found at a higher energy than the original minimum. Therefore, the results shown in $4 \mathrm{~b}$ clearly demonstrate that heating and quenching a system can lead to both lower and higher energy minima and suggest that the interconnected energy minima are separated by energy barriers of differing heights.

A number of crystal structures heated, restructured, and ultimately quenched into minima that were significantly lower in energy than the original structure. Chlorpropamide forms $\gamma$ and $\epsilon$ quenched into minima more than $10 \mathrm{~kJ} / \mathrm{mol}$ lower in energy than the original minimized structure (Figure 4). Tolbutamide form $\alpha$ and $\delta$ restructured into a minima more than $5 \mathrm{~kJ} / \mathrm{mol}$ lower in energy. This effect of significant lattice energy decrease after heating and quenching can occur in crystals of more rigid molecules as well. Pyrazinamide form $\beta$ restructured into a minimum lower in energy by $9.15 \mathrm{~kJ} / \mathrm{mol}$ as shown in previous work ${ }^{32}$ and reproduced in Figure 6b. It is interesting to note that the significant restructuring and energy changes in tolbutamide and chlorpropamide also involve a partial loss of symmetry and an increase in the number of molecules in the asymmetric unit, suggesting that the additional degrees of freedom in the supercell are in part responsible for the restructuring and decrease in energy. A further analysis of the space groups of minima in multi-minima basins is presented later in section 4.3. We note also that these restructuring events above do not necessarily suggest that these experimental forms are unstable. OPLS-AA was previously shown to give lattice energy differences in these systems that are not in precise agreement with experimentally measured enthalpy differences. ${ }^{32}$ These large changes in the lattice energy are therefore more likely a reflection of the fact that the force field is imperfect in these systems, rather than being evidence for new unobserved stable forms. Indeed, a critical insight that has emerged from the blind tests of organic crystal structure prediction is that more advanced, anisotropic or tailor-fitted force fields are often necessary for accurate a priori prediction of crystal structures. ${ }^{17}$ The examples of lattice energy decrease are high- 
lighted here to demonstrate the effect that for a given potential it is possible for a structure to become significantly lower in energy after heating and quenching even though the original structure had been completely lattice energy minimized. The effect of lattice energy minima being heated and subsequently reminimized into a lower energy configuration was shown previously in crystals of acetic acid by Mooij et al. ${ }^{28}$ This same effect observed here for polymorphs of chlorpropamide, tolbutamide, and aripiprazole, shown in Figure 4 and the Supporting Information, respectively, demonstrates that this effect can occur in crystals of larger drug-like molecules as well.

It is not trivial to take this temperature dependence and translate it into unambiguous descriptions of the roughness of the energy surface. Simple analyses assuming Arrhenius behavior are not directly valid in the solid state, especially with very low energy barriers, and the number of independent minima precludes direct counting of transitions between states. However, looking at the rates of transition between different minima in systems with a large number of minima at $300 \mathrm{~K}$, in most cases the energy of the minima are uncorrelated beyond 50 to 200 ps. Even in the more rigid pyrazinamide, the minima interconversion was seen to occur on the timescale of $50 \mathrm{ps}$ (fig. 7). This suggests that the rates among all of the different transitions for structural minima that are distinguishable in powder diffraction analysis are at least on this timescale. Kinetically, this means that the structures are not reasonably seen as distinct states, but rather as an equilibrium ensemble. An exact estimate of transition barriers between minima would require identifying the precise reaction coordinate(s) and transition state(s) connecting the minima, which is outside the scope of this work. However, fluctuations in potential energy are on the order of 3-3.5 kJ/mol per molecule at $300 \mathrm{~K}$ for the flexible molecules with multiple minima simulated in this study, suggesting that barriers are of this general magnitude. 


\section{Minima coalescence and implications for lattice-energy-based CSP studies}

The multi-minima behavior of ambient-temperature molecular crystals has a number of implications for future CSP studies based on lattice-energy minima. It is assumed in these CSP studies that each energy minimized configuration found on the lattice energy landscape corresponds with a unique structure at ambient conditions. We present in this work a number of crystal polymorphs which have a plethora of lattice energy minima that all came from the same continuous ambient temperature trajectory. The microscopic reverse of this result is that heating each of these unique minima should result in the same equilibrium ensemble rather than a set of distinct crystal structures. This strongly suggests that not all lattice energy minima correspond with a unique ambient temperature ensemble, and many distinct minima will 'coalesce' when heated.

The energy difference between two coalescing minima can be quite large. Figure 4a shows the cluster of minima for chlorpropamide $\alpha$ found from the same $300 \mathrm{~K}$ trajectory. The minima span an energy range of $2.5 \mathrm{~kJ} / \mathrm{mol}$ indicating that minima separated on the lattice energy landscape by a value at least as large as this are capable of coalescing into the same ambient temperature structure. This suggests that two minima on a CSP landscape as far apart as $2.5 \mathrm{~kJ} / \mathrm{mol}$ could ultimately correspond with 'the same' high temperature structure.

We note that although none of the snapshots from the $300 \mathrm{~K}$ trajectory of chlorpropamide $\alpha$ minimized into the original global minimum shown in Figure 4a, the phase space at $300 \mathrm{~K}$ was nevertheless reached by heating up the original lattice energy minimum and thus are examples of minima coalescing into the same high temperature structure. This global minimum is separated from the cluster of minima at $300 \mathrm{~K}$ by approximately $3.0 \mathrm{~kJ} / \mathrm{mol}$, suggesting that the energy difference between coalescing minima can be quite large.

The fact that these clusters of minima correspond to the same ambient temperature structure also highlights the importance of finding the true global energy minimum within 
the basin in order to draw meaningful conclusions about the structures rank order relative to other polymorphic forms. Experimental polymorphs on the CSP landscape can often be separated by less than $2.0 \mathrm{~kJ} / \mathrm{mol} .{ }^{11}$ The lattice energy landscapes presented here for crystals such as chlorpropamide $\alpha$ and $\beta$ show that the minimized lattice energy for an ambient temperature structure can differ by well over $2.0 \mathrm{~kJ} / \mathrm{mol}$ depending on the specific configuration that is ultimately minimized and chosen to represent the structure. In the case of chlorpropamide $\beta, \gamma$, and $\epsilon$, a more energetically stable minimum was found after heating above $200 \mathrm{~K}$, suggesting that adding a heat-quench cycle as a final refinement step to CSP could lead to more stable structures, at least in flexible and disordered crystals.

An important observation from this work is that configurations from the same finitetemperature trajectory can quench into more than one space group, and therefore presumably structures with different space groups can heat into the same ensemble. Table 1 shows the lattice parameters and space group for the two new minima of Pyrazinamide $\alpha$ found at $300 \mathrm{~K}$ as well as the original $0 \mathrm{~K}$ minimum. The three superimposed structures are shown visually in Figure 8. The original minimum as well as one of the new minima both have the $\mathrm{P} 22_{1} / \mathrm{c}$ space group symmetry. However, the third minimum found after heating at $300 \mathrm{~K}$ instead has $\mathrm{P} \overline{1}$ space group symmetry, demonstrating that minima with different space groups on the lattice energy landscape can coalesce into the same ambient temperature ensemble. This is important for the analysis and interpretation of CSP results because the usual practice is to perform structure prediction separately in each space group. The observation that structures with different space groups can occupy the same finite temperature ensemble means that CSP structure sets should be analyzed across space groups to identify such ensembles.

Crystals of the flexible compounds also quenched into minima having different space groups. Chlorpropamide form $\delta$ at $0 \mathrm{~K}$ has the space group Pbca with $\mathrm{Z}^{\prime}=1$. However, at higher temperatures above $50 \mathrm{~K}$, the trajectory also quenched into minima with space group Pca2 $2_{1}$ and $Z^{\prime}=2$. A full list of all space group conversions observed in these compounds is presented in Table 2. Additionally, all of the crystals of the flexible compounds (except 
chlorpropamide $\delta$ ) reverted from a Z' $\leq 2$ and a non-trivial space group initially into minima with the trivial P1 space group and no internal symmetry after heating to $300 \mathrm{~K}$ and quenching to $0 \mathrm{~K}$. Figure 9 depicts the overlaid structures of the 100 minima of chlorpropamide $\beta$ found after quenching from $300 \mathrm{~K}$. All 100 minima have subtle differences in the molecular position and dihedral orientations such that there is no internal space group symmetry.

The crystals of the disorder-prone compounds cyclopentane and succinonitrile also heated and quenched into minima with no symmetry at $100 \mathrm{~K}$ and $300 \mathrm{~K}$, respectively. These non-symmetric minima reflect the fact that in some crystals, particularly for the flexible molecules, there are multiple minima that are close to the expanded unit cell except with a few molecules jarred out of place enough to lose the original symmetry. It is possible that the number of such similar non-symmetric minima may give an indication of the amount of entropy that the symmetric cell will have in the ambient temperature ensemble. A full enumeration of the non-symmetric supercell minima and its correlation to $300 \mathrm{~K}$ entropy is left for future investigation.

The phenomena of high-symmetry cells devolving into a multitude of lower- or no- symmetry minima is a direct product of the fact that all MD simulations and crystal structure optimizations were performed on supercells of the experimental unit cells. The configurational space available to these supercells is greater than what is typically sampled during CSP because the constraints on translational symmetry between molecules in different unit cells are removed within the supercell. For many of the crystals exhibiting multi-minima behavior, many of the supercell minima lack complete translational symmetry between unit cell copies within a supercell. These would not have been located in usual CSP, which only samples degrees of freedom within the unit cell. Therefore, the large set of minima presented in this study suggest that real crystals have additional minima beyond just the standard overprediction problem found in CSPs constrained to unit cells. For any given experimental x-ray diffraction study, there are almost certainly structural defects or other small changes between individual unit cells that are not detected in standard diffraction. These 
undetectable perturbations could involve small random variations within a large number of unit cells, or significant deviations within a small subset of cells. However, exploring the severity of crystal perturbation that is thermally averaged away in experimental diffraction is beyond the scope of the current study.

Given the appearance of multiple minima within ambient temperature lattice energy basins, an immediate question is what segments of the molecules are the source of the conformational flexibility leading to the multi-minima behavior. To investigate this, we computed the root mean square fluctuation (RMSF) of each of the atoms among the 100 minimized structures for the five polymorphs of the three molecules with the most prevalent multi-minima behavior: aripiprazole (Fig. 10), chlorpropamide (Fig. 11), and tolbutamide (Fig. 12). In all cases, we find there is a wide variation in the atomic RMSF between polymorphs, as well as between the three molecular systems, which defies easy categorization and summary. We use the same color-RMSF scale in figures 10-12 for easier comparison of the relative structural fluctuations within polymorphs, between polymorphs, and between the three different molecular systems.

Aripiprazole has the least overall conformational variation, with a flexible ether connector between the piperazine moiety and the benzolactam, but no obvious free dihedrals. In form II (Fig. 10b) and form IV (Fig. 10d), there is very little variation among minima for these molecules, with small changes in the benzolactam moiety in form IV. In form I (Fig. 10a) and form X (Fig. 10e), the molecules undergo flexing/buckling around the ether linkage and in the benzolactam group in ways that mostly preserve the hydrogen bonding network. In form III (Fig. 10c), there are infrequent flips of the piperazine ring with respect to the dichlorinated phenyl ring, which the rest of the crystal accommodates with moderate global rearrangement.

Chlorpropamide has more conformational flexibility, particularly as a result of a freely rotating alkyl group attached to the nitrogen of the amide. Form $\gamma$ (Fig. 11c) and form $\delta$ (Fig. 11d) have little variation between minimized structures, but there are some adjust- 
ments of the alkyl groups in form $\delta$, where because of the packing, the alkyl tails have slightly more room. In forms $\alpha$ (Fig. 11a), $\beta$ (Fig. 11b), and $\epsilon$ (Fig. 11e), the alkyls have significantly more structural variability between minimized snapshots. In form $\alpha$, which has a herringbone packing with a bent molecule, the alkyl tails have some structural variability, but rearrangements are less common, only occurring in a third of the molecules, mostly independently, and with both dihedrals of the alkyl chain moving to accommodate the smaller pocket without disturbing the overall packing significantly, though there is some overall structural adjustment throughout the crystal. In form $\beta$, the alkyl groups are in a partially open pocket, away from the aromatic rings that constrain the alkyl group in forms $\gamma$ and $\delta$, but still in contact with alkyl groups in other molecules. The last dihedral in the alkyl chain is almost entirely free to rotate, with substantial movement throughout the tail. Because the pockets containing the alkyl groups are open, the aromatic rings only need to move moderately to accommodate the pocket motions. Significantly, the changes within one molecule and within a unit cell seem to be mostly independent from other molecules and cells. Finally, in form $\epsilon$, the alkyl chains are in an entirely open pocket, but in this case are not interacting with other alkyl chains, resulting in a final dihedral that is almost free to rotate, but with only moderate freedom of motion in the other dihedrals in the alkyl group, and very little conformational variability or readjustment through the rest of the molecule.

Finally, tolbutamide has an even longer alkyl chain, and all polymorphs have significant motion in the chain (Fig. 12). In forms I (Fig. 12a), III (Fig. 12c), IV (Fig. 12d, and V (Fig. 12e), there is significant motion in the alkyl chains, with some compensating motion in the rest of the crystal. Form I and form III are most similar to form $\epsilon$ of chlorpropamide, with relatively open pockets for the alkyl group formed by aromatic groups, allowing them to rotate freely, with variability in the rest of the molecule. Form I has somewhat more concerted rotation of both of the last two dihedrals and moderate overall adjustment, with form III mostly varying in the last dihedral, with the dihedrals all moving relatively independently from molecule to molecule. Form IV has alkyl groups next to each other with high mobility, 
also resulting in mobility in the neighboring aromatic groups. In contrast, form II has substantial conformational activity throughout the entire cell and is around 2-3 times as variable on average than the other polymorphs. The alkyl tails are somewhat more flexible, but all of the crystal is relatively highly structurally diverse.

In the majority of cases, this structural variation does not correlate significantly between symmetrically equivalent molecules within the supercell. There is some correlation in localized pockets, as surrounding molecules must adjust when there are changes in alkyl dihedrals, but these correlations do not appear to propagate spatially beyond the pockets. Additionally, the motions are not particularly harmonic, with all alkyl groups undergoing substantial switches between discrete dihedral torsional states, though the aripiprazole "breathing" motions might have substantial harmonic character. Finally, the number of minimized structures appears, as expected, to increase with the number of torsions that are free to rotate. Although aripiprazole has a number of dihedral torsions that themselves have low barriers, they are in a linking region between two large heterocycles, which significantly constrains their flexibility once packed.

Overall, the results in this work represent clear evidence that multi-minima behavior exists in finite-temperature crystals of standard organic small molecules. This multi-minima behavior could help explain at least some of the factors contributing to the significant overprediction of crystal structures in standard $0 \mathrm{~K}$ lattice-energy CSP studies. These CSP studies frequently produce 100s-1000s of similar-energy minima for molecules that only have 2-5 experimentally observed forms at ambient temperatures. We show here that minima separated by as much as $2-3 \mathrm{~kJ} / \mathrm{mol}$ can ultimately correspond with the same ambient temperature ensemble, including minima that have different space groups. In the cases where polymorphic over-prediction has occurred, it seems reasonable to postulate that the experimental structures have multiple corresponding lattice energy minima that are being identified and displayed in the $0 \mathrm{~K}$ CSP landscape. 


\section{Conclusions}

The multi-minima behavior of organic small molecule crystals was explored in twelve different polymorphic systems through the use of finite temperature heating followed by rapid quenching to $0 \mathrm{~K}$. Many of the finite-temperature basins contained more than one local energy minima. This contrasts with the traditional picture of crystal structure landscapes wherein each minima on the lattice energy landscape corresponds with a unique ambienttemperature structure.

Molecular flexibility and crystalline disorder were found to correlate with the crystal exhibiting multi-minima behavior. All three systems with multiple flexible degrees of freedom and both systems with dynamic disorder quenched from $300 \mathrm{~K}$ into more than one lattice energy minima. Conversely, the smaller and more rigid organic small molecules tended to quench into a single or a small number of lattice energy minima. The existence of multiple minima can also be crystal-structure-dependent. Pyrazinamide $\alpha, \beta$, and $\gamma$ were found to have more than one local energy minima, while pyrazinamide $\delta$ quenched from $300 \mathrm{~K}$ into a single unique minimum.

The number of minima found in the systems with multi-minima behavior also correlated with the simulation temperature. At higher temperatures, the system tended to find more lattice energy minima, and the minima also tended to be higher in energy than those found at low temperature. However, in certain crystals the heating and subsequent quenching of the configurations resulted in minima that were lower in energy by approximately 1-2 $\mathrm{kJ} / \mathrm{mol}$ than the minima obtained from minimizing the published experimental structure in OPLS-AA. This energy change of $1-2 \mathrm{~kJ} / \mathrm{mol}$ also highlights the dependence of the predicted stability of a crystal basin on the local energy minima used to characterize it when using 0 K CSP approaches.

The source of the conformational variability between degenerate minima appears to be primarily deriving from changes in the dihedral angles of molecules, and additional compensating changes in the rest of the crystal during the finite temperature trajectory. This is 
consistent with the observed multi-minima behavior upon minimization, as different dihedrals will minimize to different final structures. These dihedral motions usually occur within isolated pockets in the crystals, and thus occur relatively independently from one unit cell to another.

In this work, we focused specifically on multi-minima behavior in single component freeform crystals. Other crystal forms such as salts, co-crystals, and solvates are likely to have multi-minima behavior as well, particularly due to the additional soft degrees of freedom. However, this hypothesis will need to be tested.

The multi-minima behavior explored in this work represents a plausible partial explanation for some of the over-prediction of structures commonly seen in $0 \mathrm{~K}$ crystal structure prediction studies, at least in flexible and disordered crystals where multi-minima behavior is readily observed. The $300 \mathrm{~K}$ trajectories of crystals in this work quenched into as many as 100 distinct lattice energy minima, spanning a minimized lattice energy range of 2-3 kJ/mol, and corresponding to multiple different space groups. Experimental crystal polymorphs are therefore highly likely to have multi-minima behavior and are better represented by an ensemble of structures that include several or many minima rather than a single lattice-energy minimum.

\section{Acknowledgments}

Molecular simulations were performed using resources from the Extreme Science and Engineering Discovery Environment (XSEDE), which is supported by National Science Foundation grant number ACI-1548562. Specifically, it used the Bridges system, which is supported by NSF award number ACI-1445606, at the Pittsburgh Supercomputing Center (PSC). MRS and ECD's work was supported in part by NSF through the grant NSF-CBET 1351635. DPM and GMD acknowledge funding from the Engineering and Physical Sciences Research Council (EPSRC), grant number EP/K018132/1. We thank Nathan Abraham for comments on 
drafts of the paper.

\section{Tables and Figures}

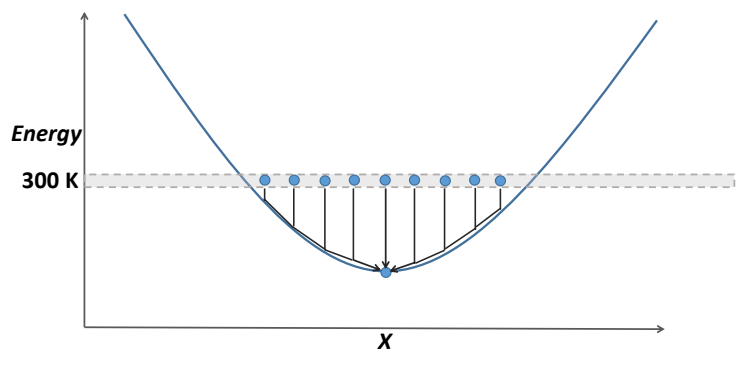

(a) Smooth basin

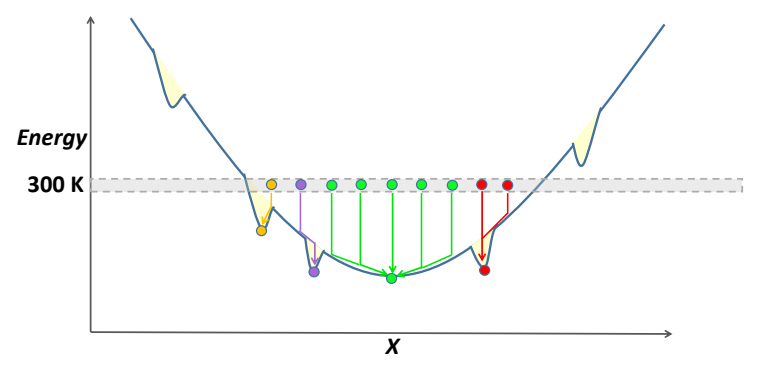

(b) Rough basin

Figure 1: Hypothetical basins which are a) smooth and contain a single energy minima or b) rough and contain multiple local energy minimum. Configurations sampled at $300 \mathrm{~K}$ in the smooth basin will always return to the same minimum after minimization. Configurations sampled in the rough basin will minimize into multiple different local minima. This is a conceptual schematic of the physical system and a simplification of the much higher dimensional energy surface. The multiple minima within real basins will likely exhibit a diversity of well depths and widths. 


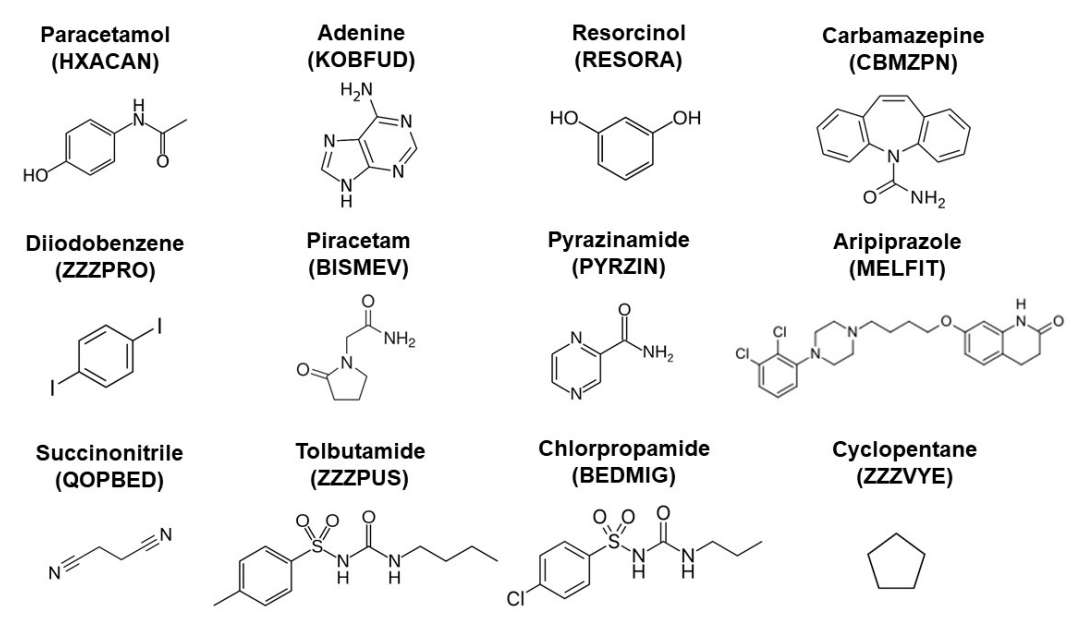

Figure 2: All twelve organic small molecule compounds examined in this work along with the corresponding CSD refcodes.

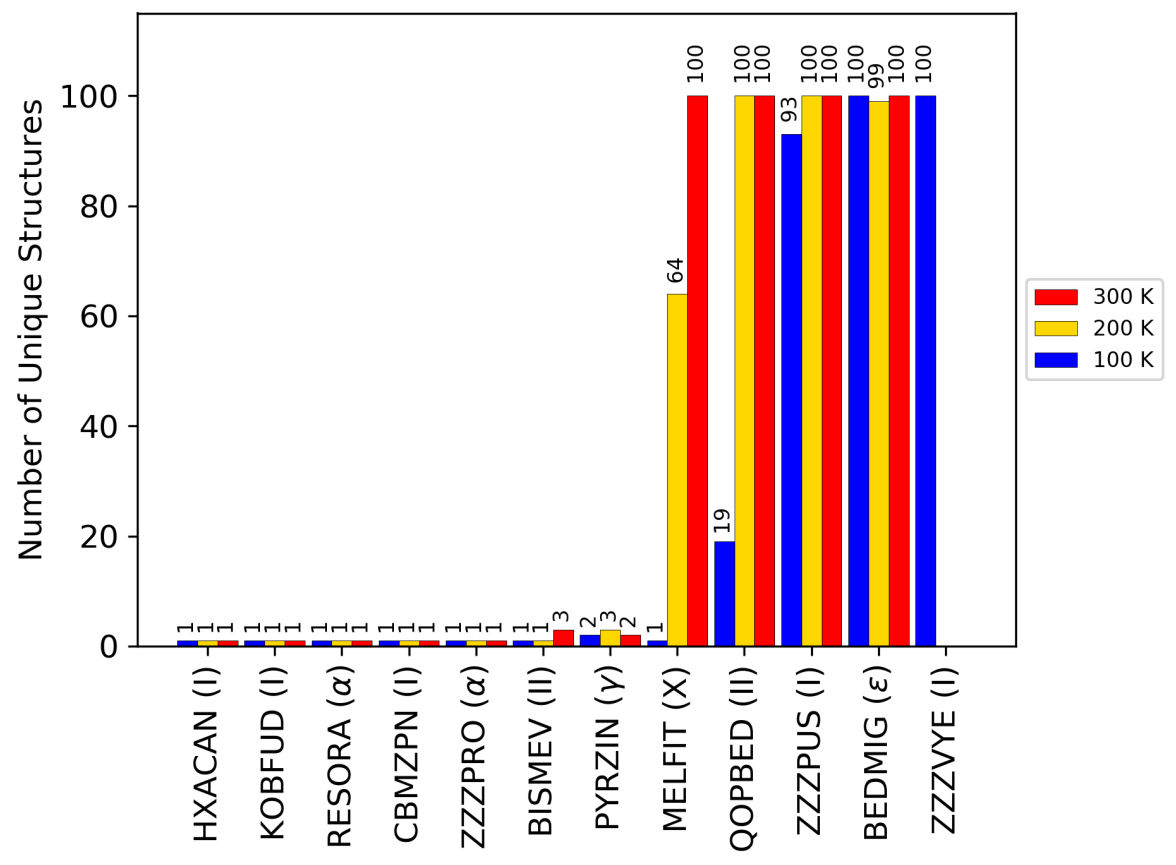

Figure 3: Crystal structures of 8 of the 12 systems exhibited multi-minima behavior after quenching $5 \mathrm{~ns}$ trajectories run at $300 \mathrm{~K}, 200 \mathrm{~K}$, and $100 \mathrm{~K}$. The polymorph with the highest number of minima at $300 \mathrm{~K}$ was used to represent each compound. The systems which had more than 1 minimum at $300 \mathrm{~K}$ tended to be the larger and more flexible molecules, or molecules with dynamically disordered polymorphs. The number of minima found after quenching also tended to be larger at higher temperatures. 


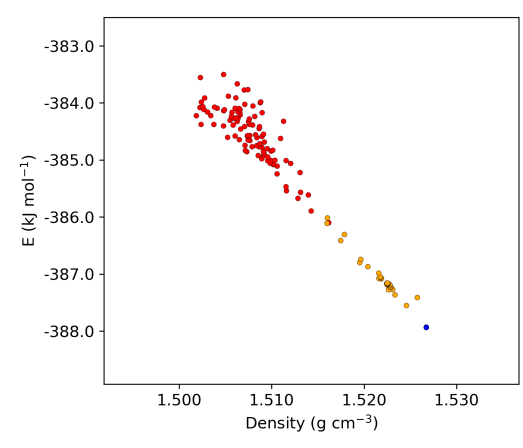

(a) Form $\alpha$

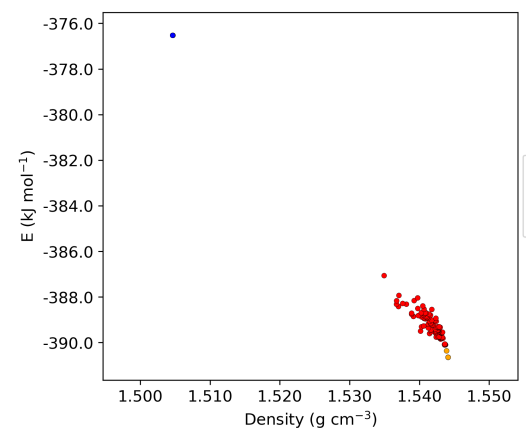

(c) Form $\gamma$

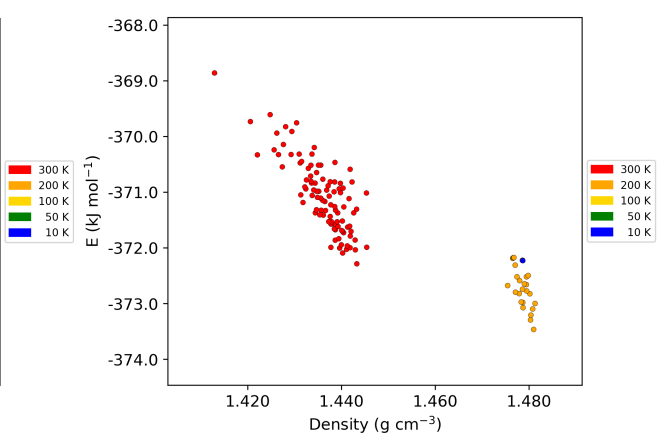

(b) Form $\beta$

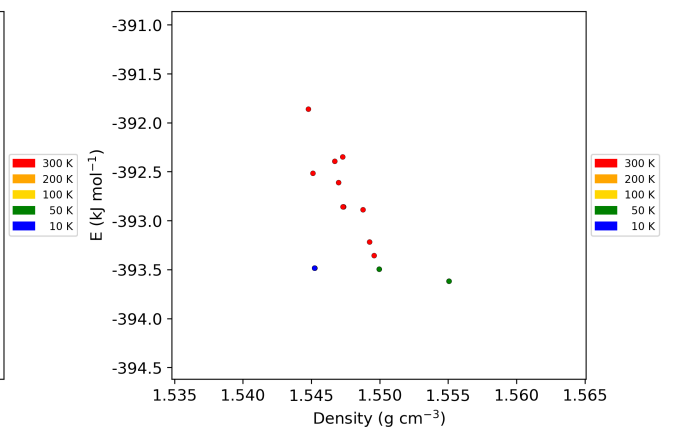

(d) $\operatorname{Form} \delta$

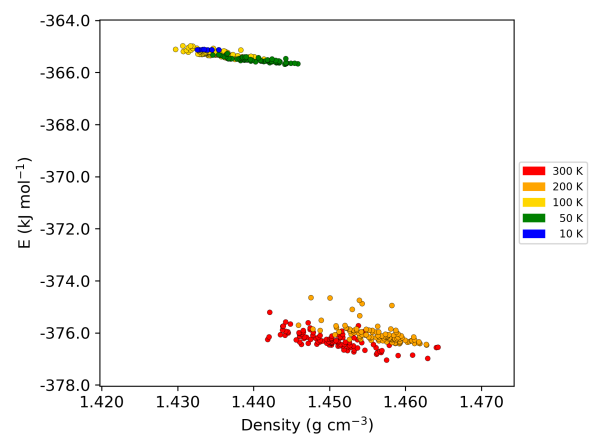

(e) Form $\epsilon$

Figure 4: Lattice energy minima for chlorpropamide a) $\alpha$ b) $\beta$ c) $\gamma$ d) $\delta$, and e) $\epsilon$ found from quenching the trajectories at various temperatures. Each lattice energy minima is colored by the lowest temperature simulation which generated it. Forms $\alpha-\gamma$ and $\epsilon$ both found 100 unique minima at $300 \mathrm{~K}$, while form $\delta$ found just 13 . The number of minima found after quenching is larger at higher temperatures. Form $\gamma$ and $\epsilon$ also experienced a significant restructuring into a lower energy region of the local crystal basin when heated and quenched above $100 \mathrm{~K}$. 


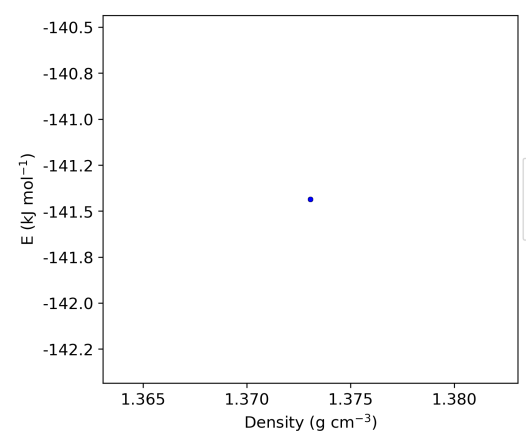

(a) Form I

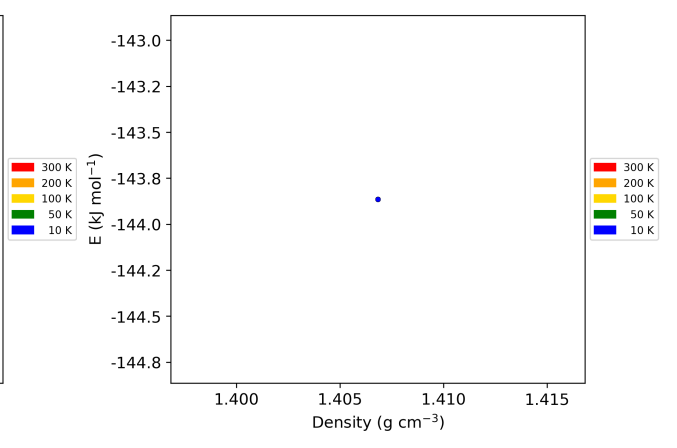

(b) Form III

Figure 5: Lattice energy minima for carbamazepine a) form I and b) form III found from quenching the trajectories at various temperatures. Each lattice energy minima is colored by the lowest temperature simulation which generated it. Both form I and form III quenched back to the original lattice energy minima after heating up to $300 \mathrm{~K}$.

Table 1: Lattice parameters (in nm), angles (in degrees), and space group symmetry for the three minima found from quenching the finite temperature simulations of pyrazinamide $\alpha$. Minimum 1 corresponds with the structure obtained from minimizing the original experimental structure as well as from quenching simulations at $100 \mathrm{~K}$ and below. Minimum 2 is found after quenching configurations sampled at $200 \mathrm{~K}$. Minimum 3 is found from quenching configurations sampled at $300 \mathrm{~K}$.

\begin{tabular}{lccc}
\hline \hline Lattice Parameter & Minimum 1 & Minimum 2 & Minimum 3 \\
\hline $\mathrm{a}$ & 2.308 & 2.187 & 2.228 \\
$\mathrm{~b}$ & 2.798 & 2.732 & 2.774 \\
$\mathrm{c}$ & 2.296 & 2.281 & 2.273 \\
$\alpha$ & 90 & 90 & 90 \\
$\beta$ & 113 & 95 & 104 \\
$\gamma$ & 90 & 90 & 93 \\
Space group & $\mathrm{P} 21 / \mathrm{c}$ & $\mathrm{P} 2_{1} / \mathrm{c}$ & $\mathrm{P} \overline{1}$
\end{tabular}




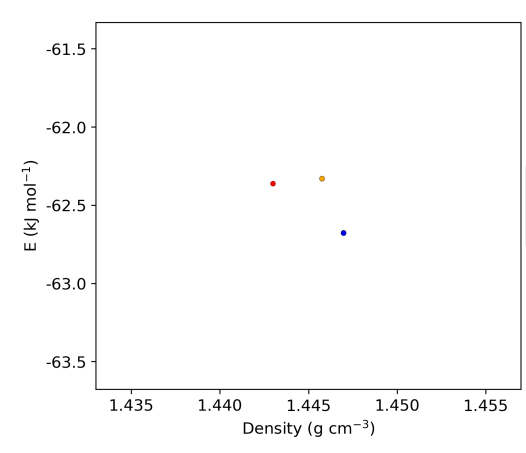

(a) Form $\alpha$

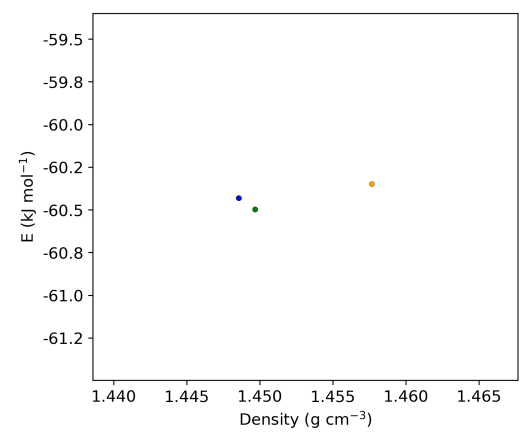

(c) Form $\gamma$

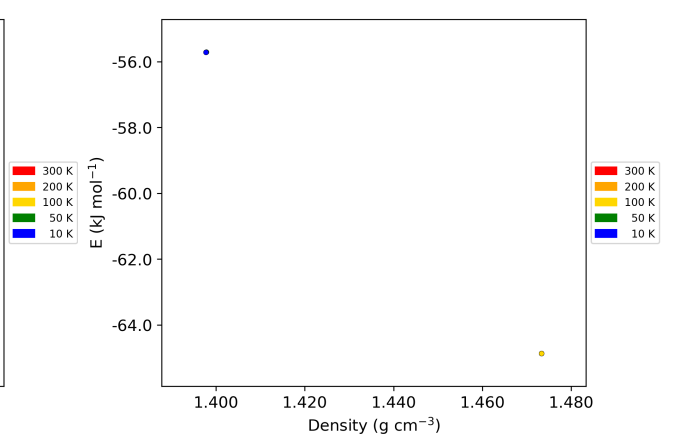

(b) Form $\beta$

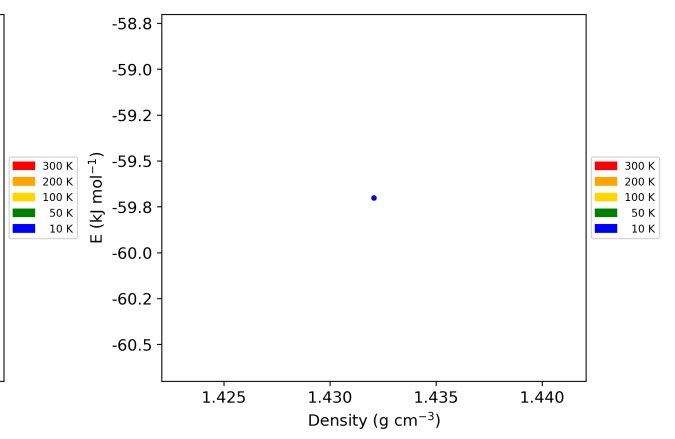

(d) Form $\delta$

Figure 6: Lattice energy minima for pyrazinamide a) $\alpha$ b) $\beta$ c) $\gamma$ and d) $\delta$ found from quenching the trajectories at various temperatures. Each lattice energy minima is colored by the lowest temperature simulation which generated it. The polymorphs of pyrazinamide demonstrate that multi-minima behavior at $300 \mathrm{~K}$ is crystal-structure-dependent. Pyrazinamide a) $\alpha$ and c) $\gamma$ quenched into 3 and 2 minima after sampling at $300 \mathrm{~K}$. Conversely, pyrazinamide b) $\beta$ and d) $\delta$ always quenched into a single minimum after sampling at $300 \mathrm{~K}$. Pyrazinamide $\beta$ experienced a restructuring above $50 \mathrm{~K}$ and found a new minima with a significantly lower lattice energy.

Table 2: Many crystal structures found lattice energy minima with symmetry distinct from the original minima after heating and quenching back to zero Kelvin. The original symmetry indicates the space group symmetry of the experimental structure. The additional symmetry indicates the space group symmetry of minima found for this crystal after quench which is different than the original lattice energy minimum.

\begin{tabular}{lcc}
\hline \hline Crystal Structure & Original Symmetry & Additional Symmetry \\
\hline Chlorpropamide $\delta$ & Pbca Z'=1 & Pca2 $Z_{1} Z^{\prime}=2$ \\
Chlorpropamide $\epsilon$ & Pna2 $Z^{\prime}=1$ & $\mathrm{P} 2_{1} Z^{\prime}=8$ \\
Tolbutamide $\alpha$ & $\mathrm{Pna2}_{1} Z^{\prime}=1$ & $\mathrm{P} 2_{1} Z^{\prime}=4$ \\
Tolbutamide $\delta$ & $\mathrm{P} 2_{1} / c Z^{\prime}=1$ & $\mathrm{P} 1 Z^{\prime}=2$ \\
Tolbutamide $\epsilon$ & $\mathrm{Pbcn} Z^{\prime}=1$ & $\mathrm{Pca} 2_{1} Z^{\prime}=4$ \\
Pyrazinamide $\alpha$ & $\mathrm{P} 2_{1} / \mathrm{c} Z^{\prime}=1$ & $\mathrm{P} 1 Z^{\prime}=2$ \\
1,4-Diiodobenzene $\beta$ & $\mathrm{Pccn} Z^{\prime}=0.5$ & $\mathrm{P} 2_{1} / \mathrm{c} Z^{\prime}=2$
\end{tabular}



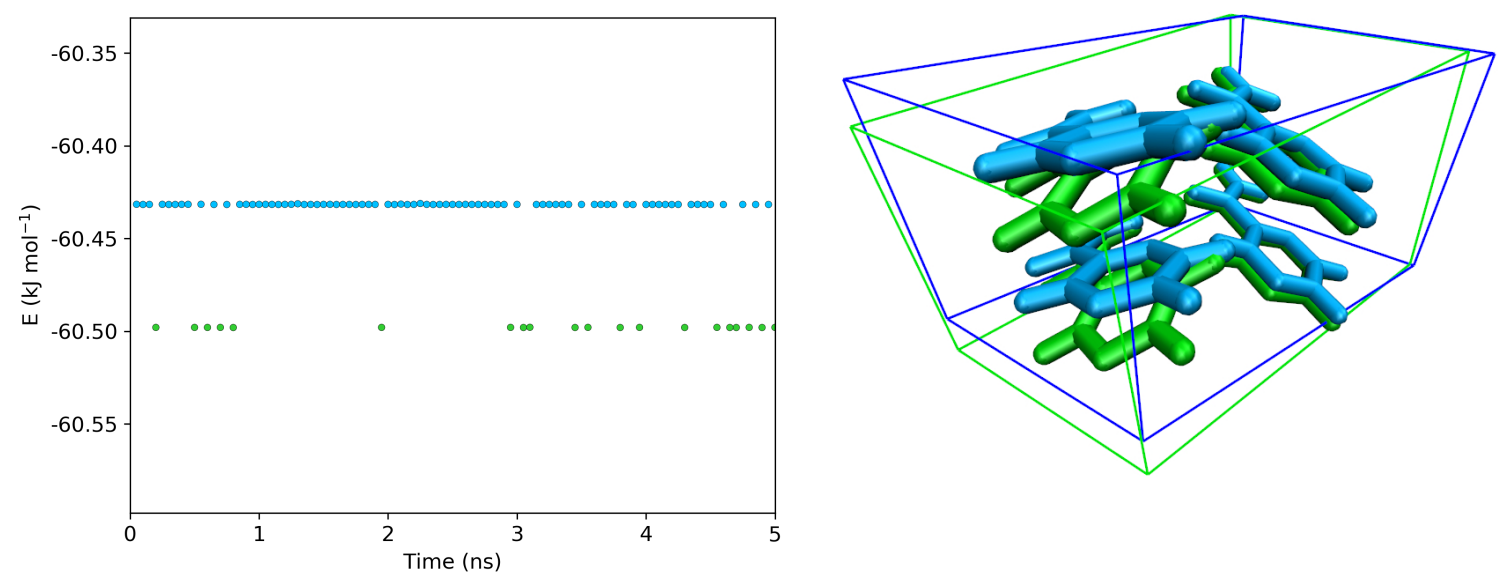

Figure 7: The sampled configurations of pyrazinamide form $\gamma$ at $300 \mathrm{~K}$ quench into two different lattice energy minima with different energies. The frequency of quenching into either minima is essentially independent of time, demonstrating that the phase spaces which quench into each minima are both sampled at equilibrium. The original minimum (blue) has Pc space group symmetry, while the new lower energy minimum (green) is in the P1 space group.

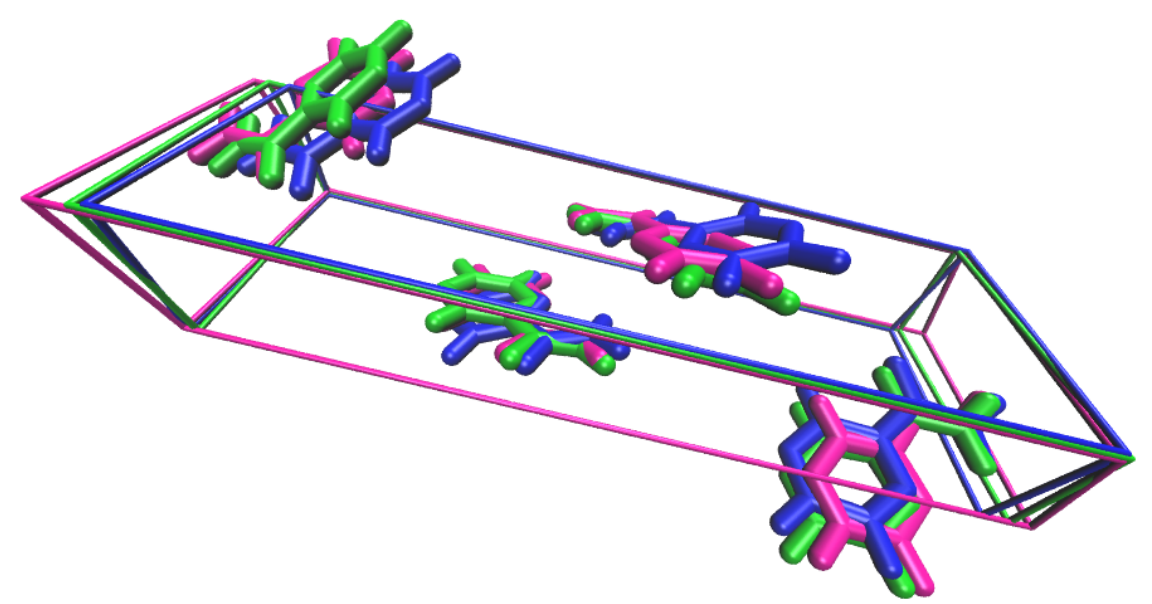

Figure 8: The lattice energy minima of pyrazinamide form $\alpha$ found after minimizing configurations from the $300 \mathrm{~K}$ trajectory have different structures and space groups symmetries. The original minimum (magenta) and one of the new minima (green) have $\mathrm{P} 21 /$ c space group symmetry. The third minimum (blue) has $\mathrm{P} \overline{1}$ space group symmetry. 


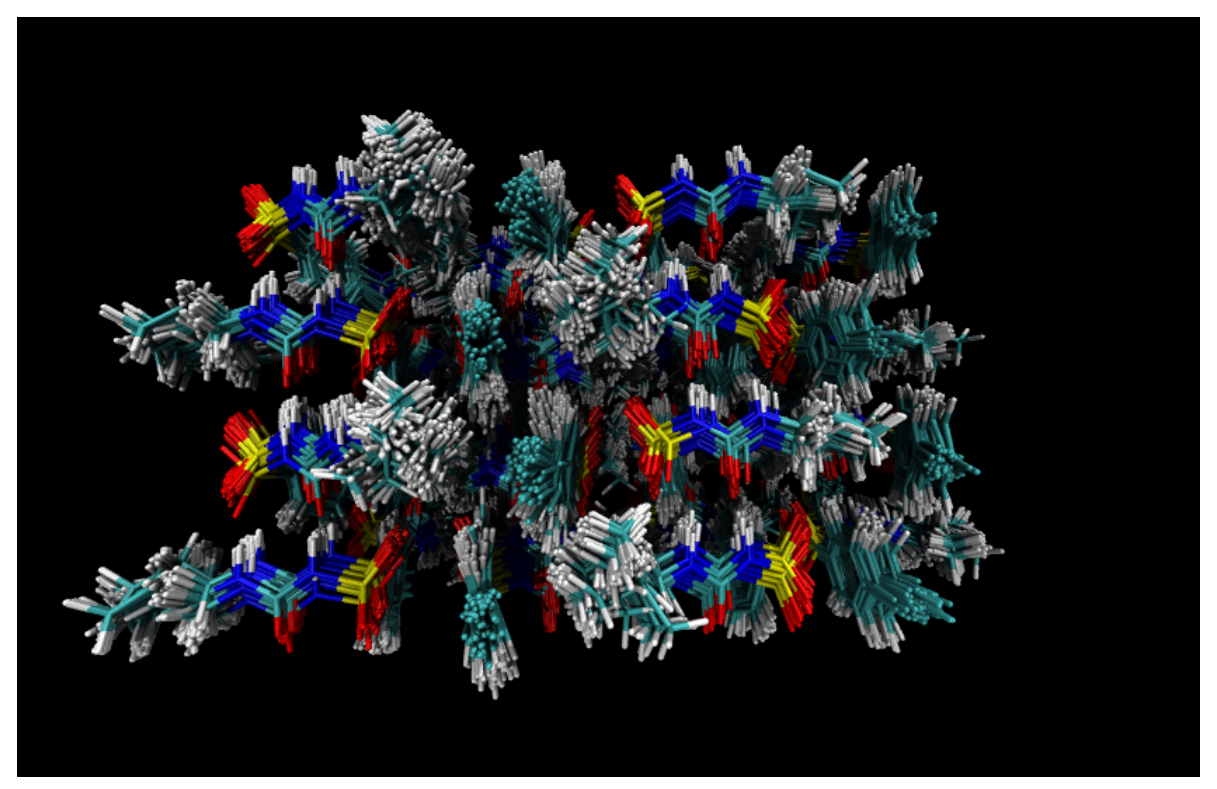

Figure 9: The numerous lattice energy minima of chlorpropamide form $\beta$ found after minimizing configurations from the $300 \mathrm{~K}$ trajectory all have subtle differences in the molecular position and dihedral orientations. All 100 structures are in the trivial P1 space group. 

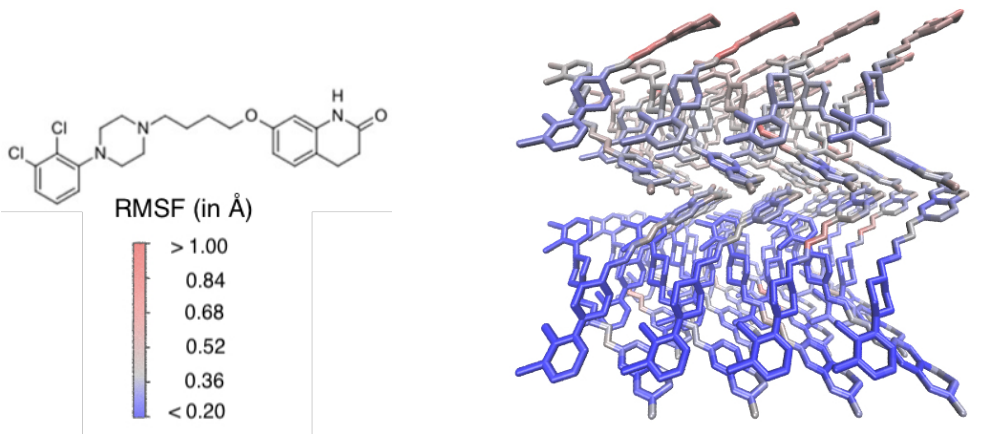

(a) Form I

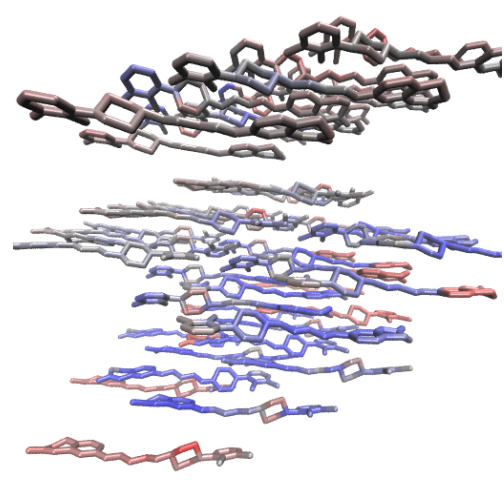

(c) Form III

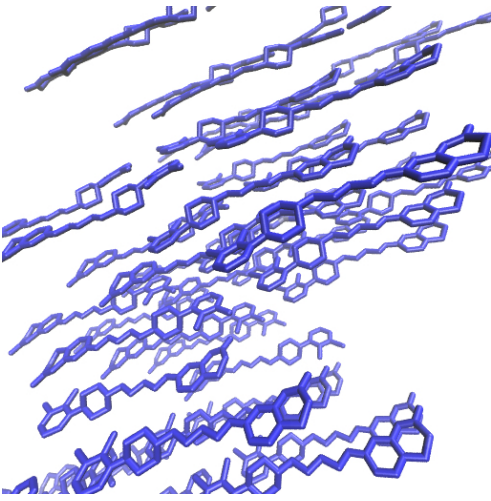

(d) Form IV

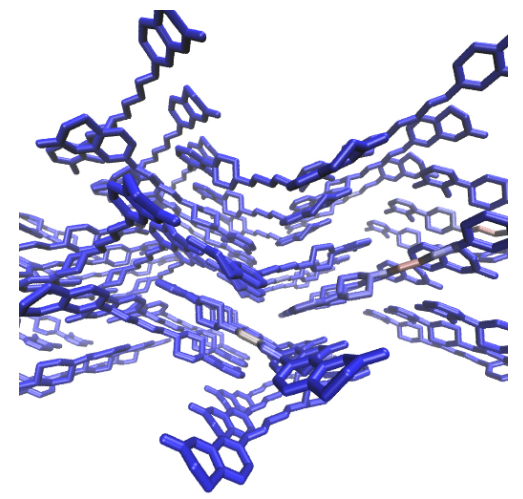

(b) Form II

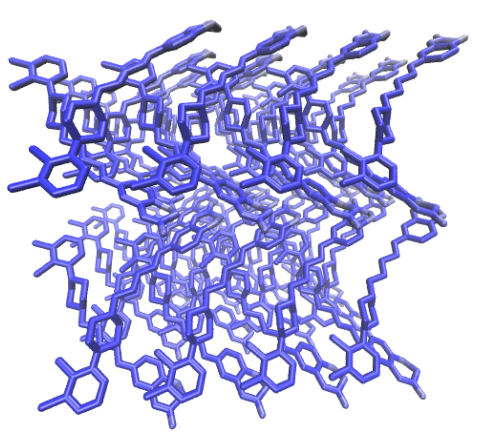

(e) Form X

Figure 10: RMSF over minimized trajectory snapshots for various forms of aripiprazole, with blue the least mobile (RMSF $<0.2 \AA$ ) and red the most mobile ( $\mathrm{RMSF}>1.0 \AA$ ), visualized on the OPLS-minimized structure from the corresponding experimental structure. Color bars are identical between this figure and those for chlorpropamide (Fig. 11) and tolbutamide (Fig. 12) for purposes of comparison. The structural heterogeneity is described in more detail in the main text. 


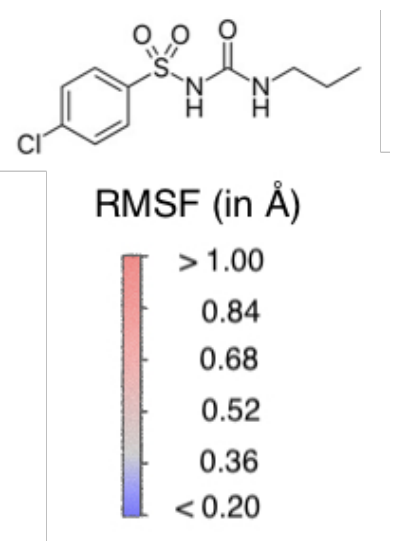

$\left[\begin{array}{r}>1.00 \\ 0.84 \\ 0.68 \\ 0.52 \\ 0.36 \\ <0.20\end{array}\right.$

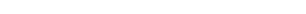

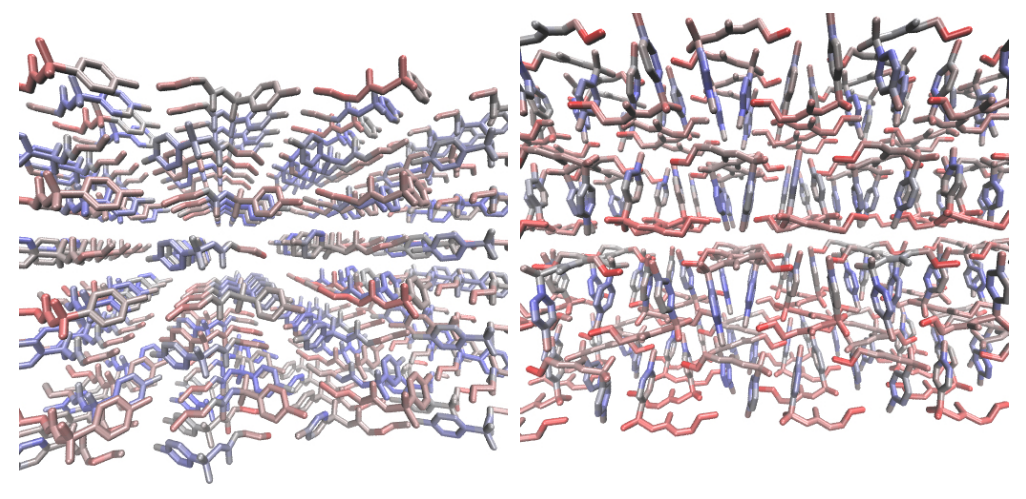

(a) form $\alpha$

(b) form $\beta$

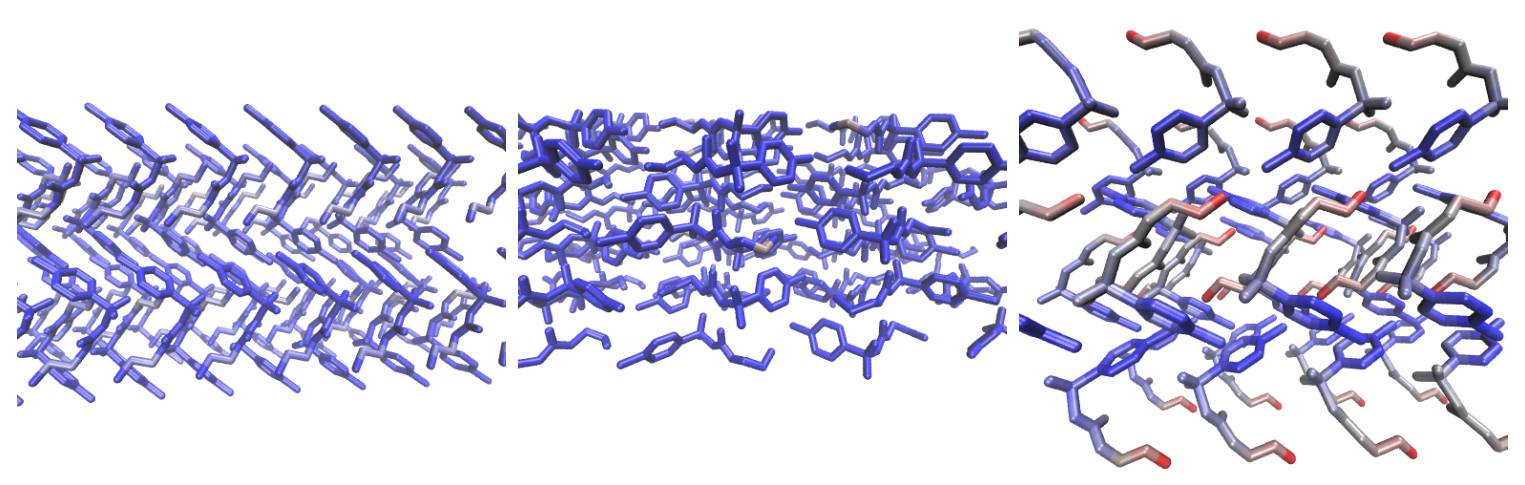

(c) form $\gamma$

(d) form $\delta$

(e) form $\epsilon$

Figure 11: RMSF over minimized trajectory snapshots for various forms of chlorpropamide, with blue the least mobile (RMSF $<0.2 \AA$ ) and red the most mobile (RMSF $>1.0 \AA$ ), visualized on the OPLS-minimized structure from the corresponding experimental structure. Color bars are identical between this figure and those for aripiprazole (Fig. 10) and tolbutamide (Fig. 12) for purposes of comparison. The structural heterogeneity is described in more detail in the main text. 

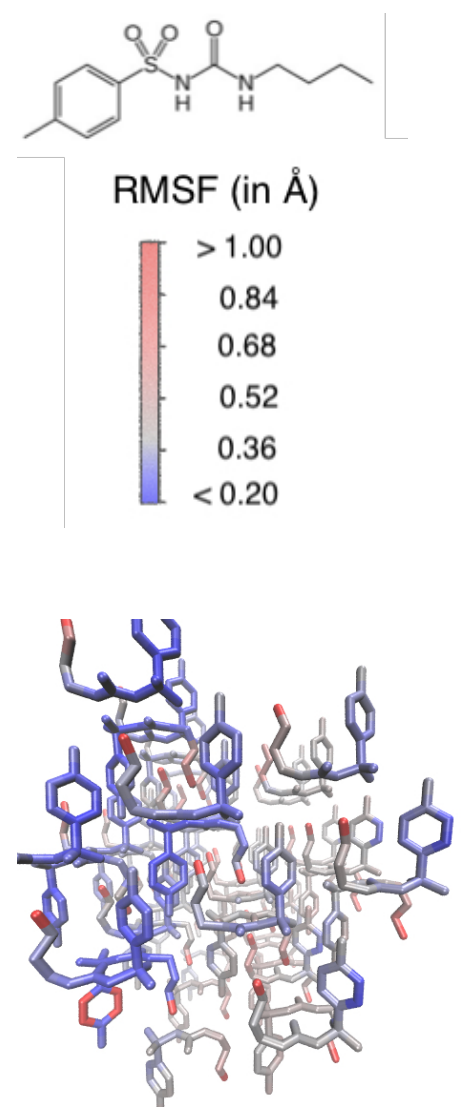

(c) form III

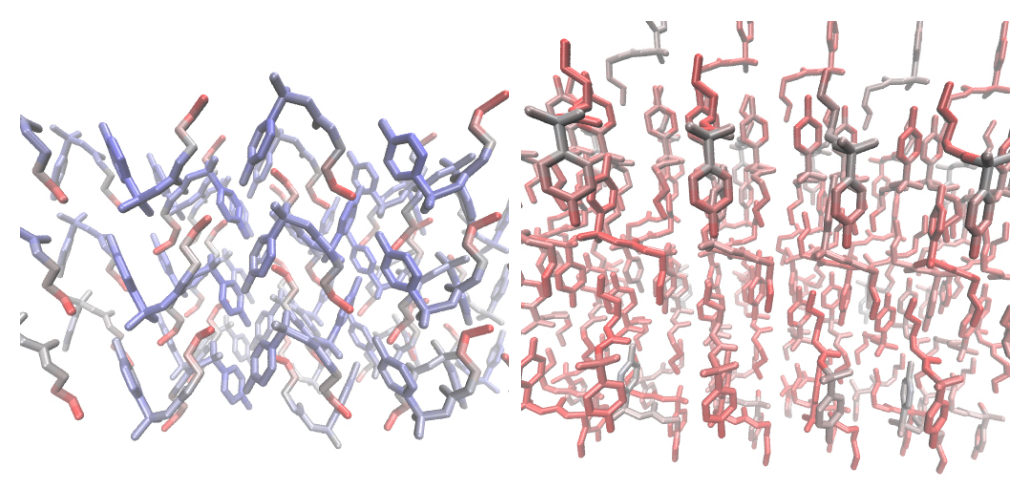

(a) form I

(b) form II

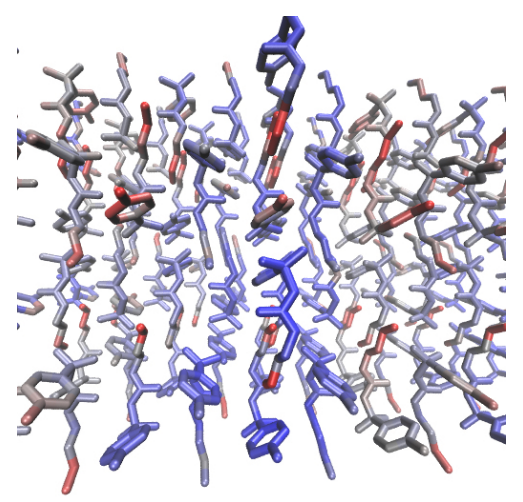

(d) form IV

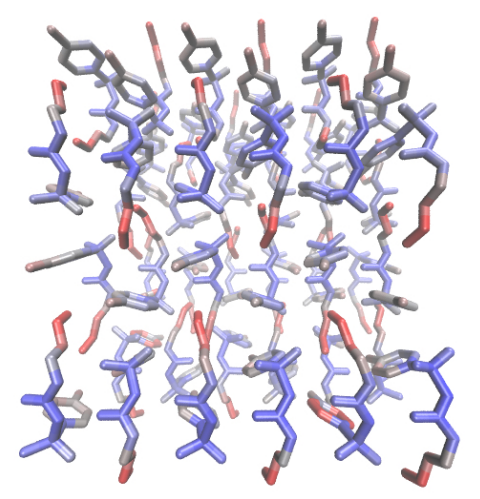

(e) form $\mathrm{V}$

Figure 12: RMSF over minimized trajectories for various forms of tolbutamide, with blue the least mobile (RMSF $<0.2 \AA$ ) and red the most mobile $(\mathrm{RMSF}>1.0 \AA)$, visualized on the OPLS-minimized structure from the corresponding experimental structure. Color bars are identical between this figure and those for chlorpropamide (Fig. 11) and aripiprazole (Fig. 10) for purposes of comparison. The form IV RMSF is collected over only the last 70 structures, as the box vectors readjusted at that point. The structural heterogeneity is described in more detail in the main text. 


\section{References}

(1) Price, S. L.; Braun, D. E.; Reutzel-Edens, S. M. Can computed crystal energy landscapes help understand pharmaceutical solids? Chem. Commun. 2016,

(2) Price, S. L.; Reutzel-Edens, S. M. The potential of computed crystal energy landscapes to aid solid-form development. Drug Discov. Today 2016, 21.

(3) Pudipeddi, M.; Serajuddin, A. T. Trends in solubility of polymorphs. J. Pharm. Sci. 2005, 94, 929-939.

(4) De Villiers, M. M.; van der Watt, J. G.; Lötter, A. P. Kinetic study of the solid-state photolytic degradation of two polymorphic forms of furosemide. Int. J. Pharm. 1992, $88,275-283$.

(5) Velaga, S. P.; Vangala, V. R.; Basavoju, S.; Boström, D. Polymorphism in acesulfame sweetener: structureproperty and stability relationships of bending and brittle crystals. Chem. Commun. 2010, 46, 3562.

(6) Trauffer, D. I.; Maassel, A. K.; Snyder, R. C. Non-Needle-like Morphology of $\beta$-Glycine Particles Formed from Water Solutions via Monodisperse Droplet Evaporation. Cryst. Growth Des. 2016, 16, 1917-1922.

(7) Chemburkar, S. R. et al. Dealing with the Impact of Ritonavir Polymorphs on the Late Stages of Bulk Drug Process Development. Org. Process Res. Dev. 2000, 4, 413-417.

(8) Chekal, B. P.; Campeta, A. M.; Abramov, Y. A.; Feeder, N.; Glynn, P. P.; Mclaughlin, R. W.; Meenan, P. A.; Singer, R. A. The Challenges of Developing an API Crystallization Process for a Complex Polymorphic and Highly Solvating System. Part I. Org. Process Res. Dev. 2009, 1327-1337.

(9) Rietveld, I. B.; Ceolin, R. Rotigotine: Unexpected Polymorphism with Predictable Overall Monotropic Behavior. J. Pharm. Sci. 2015, 104, 4117-4122. 
(10) Day, G. M. Current approaches to predicting molecular organic crystal structures. Crystallogr. Rev. 2011, 17, 3-52.

(11) Nyman, J.; Day, G. M. Static and lattice vibrational energy differences between polymorphs. CrystEngComm 2015, 17, 5154-5165.

(12) Day, G. M.; Chisholm, J.; Shan, N.; Motherwell, W. D. S.; Jones, W. An assessment of lattice energy minimization for the prediction of molecular organic crystal structures. Cryst. Growth Des. 2004, 4, 1327-1340.

(13) Day, G. M. et al. A third blind test of crystal structure prediction. Acta Crystallogr. B. 2005, 61, 511-527.

(14) Kazantsev, A. V.; Karamertzanis, P. G.; Adjiman, C. S.; Pantelides, C. C. Efficient handling of molecular flexibility in lattice energy minimization of organic crystals. $J$. Chem. Theory Comput. 2011, \%, 1998-2016.

(15) Price, S. L. Predicting crystal structures of organic compounds. Chem. Soc. Rev. 2014, 43, 2098-111.

(16) Karamertzanis, P. G.; Raiteri, P.; Parrinello, M.; Leslie, M.; Price, S. L. The thermal stability of lattice-energy minima of 5-fluorouracil: metadynamics as an aid to polymorph prediction. J. Phys. Chem. B 2008, 112, 4298-4308.

(17) Day, G. M. et al. Significant progress in predicting the crystal structures of small organic molecules-a report on the fourth blind test. Acta Crystallogr. B. 2009, 65, 107-25.

(18) Bardwell, D. A. et al. Towards crystal structure prediction of complex organic compounds-a report on the fifth blind test. Acta Crystallogr. B. 2011, 67, 535-51.

(19) Reilly, A. M. et al. Report on the sixth blind test of organic crystal structure prediction methods. Acta Crystallogr. Sect. B Struct. Sci. Cryst. Eng. Mater. 2016, 72, 439-459. 
(20) Anghel, A. T.; Day, G. M.; Price, S. L. A study of the known and hypothetical crystal structures of pyridine: why are there four molecules in the asymmetric unit cell? CrystEngComm 2002, 4, 348-355.

(21) Price, S. Why don't we find more polymorphs? Acta Crystallogr. Sect. B 2013, 69, 313-328.

(22) Price, S. L. Is zeroth order crystal structure prediction (CSP_0) coming to maturity? What should we aim for in an ideal crystal structure prediction code? Faraday Discuss. 2018 ,

(23) Vasileiadis, M.; Pantelides, C. C.; Adjiman, C. S. Prediction of the crystal structures of axitinib, a polymorphic pharmaceutical molecule. Chem. Eng. Sci. 2015, 121, 60-76.

(24) Ouvrard, C.; Price, S. L. Toward Crystal Structure Prediction for Conformationally Flexible Molecules: The Headaches Illustrated by Aspirin. Cryst. Growth Des. 2004, 4, $1119-1127$.

(25) Cabeza, A. J. C.; Day, G. M.; Motherwell, W. D. S.; Jones, W. Amide Pyramidalization in Carbamazepine- A Flexibility Problem in Crystal Structure Prediction. Cryst. Growth Des. 2006, 6, 1858-1866.

(26) Day, G. M.; S Motherwell, W. D.; Jones, W. A strategy for predicting the crystal structures of flexible molecules: the polymorphism of phenobarbital. Phys. Chem. Chem. Phys. 2007, 9, 1693-1704.

(27) Habgood, M. Form II caffeine: A case study for confirming and predicting disorder in organic crystals. Cryst. Growth Des. 2011, 11, 3600-3608.

(28) Mooij, W.; van Eijck, B.; Price, S.; Verwer, P.; Kr Loon, J. Crystal Structure Predictions for Acetic Acid. J Comp. Chem. 1998, 19, 459-474. 
(29) Raiteri, P.; Martonák, R.; Parrinello, M. Exploring polymorphism: the case of benzene. Angew. Chem. Int. Ed. Engl. 2005, 44, 3769-3773.

(30) Zykova-Timan, T.; Raitei, P.; Parrinello, M. Investigating the polymorphism in PR179: A combined crystal structure prediction and metadynamics study. J. Phys. Chem. B 2008, 112, 13231-13237.

(31) Nyman, J.; Day, G. M. Modelling temperature-dependent properties of polymorphic organic molecular crystals. Phys. Chem. Chem. Phys. 2016, 18, 31132-31143.

(32) Dybeck, E. C.; Abraham, N. S.; Schieber, N. P.; Shirts, M. R. Capturing Entropic Contributions to Temperature-Mediated Polymorphic Transformations Through Molecular Modeling. Cryst. Growth Des. 2017, 17, 1775-1787.

(33) Pronk, S.; Páll, S.; Schulz, R.; Larsson, P.; Bjelkmar, P.; Apostolov, R.; Shirts, M. R.; Smith, J. C.; Kasson, P. M.; van der Spoel, D.; Hess, B.; Lindahl, E. GROMACS 4.5: a high-throughput and highly parallel open source molecular simulation toolkit. Bioinformatics 2013, 29, 845-54.

(34) Jorgensen, W. L.; Jorgensen, W. L.; Maxwell, D. S.; Maxwell, D. S.; Tirado-Rives, J.; Tirado-Rives, J. Development and Testing of the OLPS All-Atom Force Field on Conformational Energetics and Properties of Organic Liquids. J. Am. Chem. Soc. 1996, $118,11225-11236$.

(35) Jorgensen, W. L.; McDonald, N. A. Development of an all-atom force field for heterocycles. Properties of liquid pyridine and diazenes. J. Mol. Struct. THEOCHEM 1998, 424, 145-155.

(36) Kaminski, G. A.; Friesner, R. A.; Tirado-rives, J.; Jorgensen, W. L. Comparison with Accurate Quantum Chemical Calculations on Peptides . J. Phys. Chem. B 2001, 105, $6474-6487$. 
(37) Goga, N.; Rzepiela, A. J.; de Vries, A. H.; Marrink, S. J.; Berendsen, H. J. C. Efficient Algorithms for Langevin and DPD Dynamics. J. Chem. Theory Comput. 2012, 8, $3637-3649$.

(38) Parrinello, M.; Rahman, A. Polymorphic Transitions in Single Crystals: a New Molecular Dynamics Method. J. Appl. Phys. 1981, 52, 7182-7190.

(39) Wennberg, C. L.; Murtola, T.; Hess, B.; Lindahl, E. Lennard-Jones lattice summation in bilayer simulations has critical effects on surface tension and lipid properties. $J$. Chem. Theory Comput. 2013, 9, 3527-3537.

(40) Ponder, J. W. TINKER Molecular Modeling Package. 2015.

(41) Spek, A. L. Structure validation in chemical crystallography. Acta Crystallogr. Sect. D Biol. Crystallogr. 2009, 65, 148-155.

(42) Ong, S. P.; Richards, W. D.; Jain, A.; Hautier, G.; Kocher, M.; Cholia, S.; Gunter, D.; Chevrier, V. L.; Persson, K. A.; Ceder, G. Python Materials Genomics (pymatgen): A robust, open-source python library for materials analysis. Comput. Mater. Sci. 2013, $68,314-319$.

(43) Togo, A.; Tanaka, I. Spglib: a software library for crystal symmetry search. 2018, 1-11.

(44) Torrisi, A.; Leech, C. K.; Shankland, K.; David, W. I. F.; Ibberson, R. M.; BenetBuchholz, J.; Boese, R.; Leslie, M.; Catlow, C. R. A.; Price, S. L. Solid phases of cyclopentane: combined experimental and simulation study. J. Phys. Chem. B 2008, 112, 3746-58.

(45) Hore, S.; Dinnebier, R.; Wen, W.; Hanson, J.; Maier, J. Structure of plastic crystalline succinonitrile: High-resolution in situ powder diffraction. Zeitschrift fur Anorg. und Allg. Chemie 2009, 635, 88-93. 
(46) Santiso, E. E.; Trout, B. L. A general set of order parameters for molecular crystals. J. Chem. Phys. 2011, 134, 064109.

(47) Cuendet, M. a.; Tuckerman, M. E. Free Energy Reconstruction from Metadynamics or Adiabatic Free Energy Dynamics Simulations. J. Chem. Theory Comput. 2014, 10, 2975-2986.

(48) Yu, T.-Q.; Chen, P.-Y.; Chen, M.; Samanta, A.; Vanden-Eijnden, E.; Tuckerman, M. Order-parameter-aided temperature-accelerated sampling for the exploration of crystal polymorphism and solid-liquid phase transitions. J. Chem. Phys. 2014, 140, 214109.

(49) Chen, M.; Yu, T.-Q.; Tuckerman, M. E. Locating landmarks on high-dimensional free energy surfaces. Proc. Natl. Acad. Sci. 2015, 112, 3235-3240.

(50) Steinhardt, P. J.; Nelson, D. R.; Ronchetti, M. Bond-orientational order in liquids and glasses. Phys. Rev. B 1983, 28, 784-805. 
For Table of Contents Only:

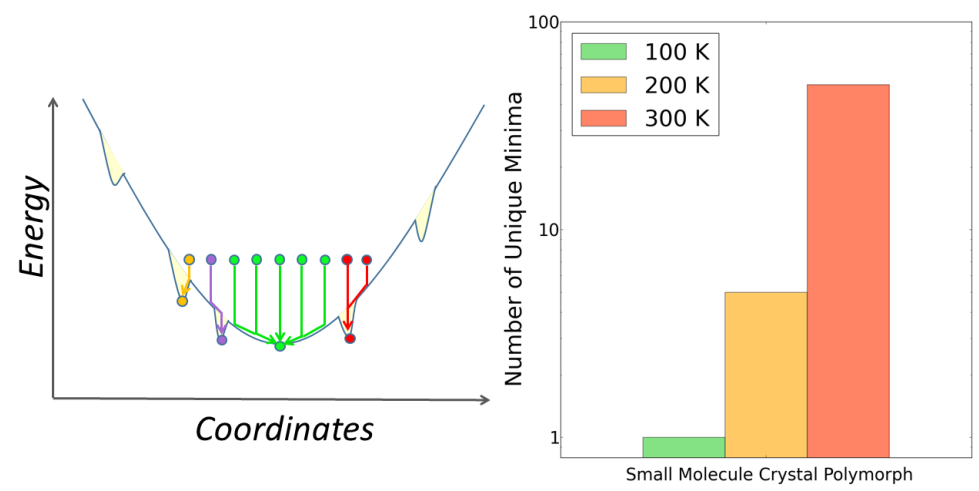

